\title{
Peptide YY3-36 Decreases Reinstatement of High-Fat Food Seeking during Dieting in a Rat Relapse Model
}

\author{
Udi E. Ghitza, ${ }^{\star}$ Sunila G. Nair, ${ }^{\star}$ Sam A. Golden, Sarah M. Gray, Jamie L. Uejima, Jennifer M. Bossert, and Yavin Shaham \\ Behavioral Neuroscience Branch, National Institute on Drug Abuse, Intramural Research Program, National Institutes of Health, Department of Health and \\ Human Services, Baltimore, Maryland 21224
}

\begin{abstract}
A major problem in treating obesity is high rates of relapse to maladaptive food-taking habits during dieting. This relapse is often provoked by acute re-exposure to palatable food, food-associated cues, or stress. We used a reinstatement model, commonly used to study relapse to abused drugs, to explore the effect of peptide YY3-36 (PYY3-36) on reinstatement of high-fat (35\%, $45 \mathrm{mg}$ pellets) food seeking induced by acute exposure to the pellets (pellet priming), a cue previously associated with pellet delivery (pellet cue), or yohimbine $(2 \mathrm{mg} / \mathrm{kg}$, a pharmacological stressor). Rats were placed on a restricted diet (16 $\mathrm{g}$ of chow per day) and lever-pressed for the pellets for 9-12 sessions (6 h/d, every $48 \mathrm{~h}$ ); pellet delivery was paired with a tone-light cue. They were then given 10-20 extinction sessions wherein lever presses were not reinforced with the pellets and subsequently tested for reinstatement of food seeking. Systemic PYY3-36 injections $(100-200 \mu \mathrm{g} / \mathrm{kg}$ ) decreased pellet priming- and pellet cue-induced reinstatement of food seeking but not yohimbine-induced reinstatement. Arcuate nucleus (Arc) injections of PYY3-36 (0.4 $\mu \mathrm{g}$ per side) decreased pellet priming-induced reinstatement. The attenuation of pellet priming-induced reinstatement by systemic PYY3-36 was reversed by systemic $(2 \mathrm{mg} / \mathrm{kg})$ but not $\operatorname{Arc}(0.5 \mu \mathrm{g}$ per side) injections of the Y2 receptor antagonist BIIE0246. Arc PYY3-36 injections did not decrease pellet cue-induced reinstatement. Finally, systemic PYY3-36 injections had minimal effects on ongoing food self-administration or heroin priming- or heroin cue-induced reinstatement of heroin seeking. These data identify an effect of systemic PYY3-36 on relapse to food seeking that is independent of Y2 receptor activation in Arc and suggest that PYY3-36 should be considered for the treatment of relapse to maladaptive food-taking habits during dieting.
\end{abstract}

Key words: arcuate nucleus; heroin self-administration; peptide YY; reinstatement; relapse; stress; Y2 receptors; yohimbine

\section{Introduction}

A main problem in dietary treatment of excessive eating is high rates of relapse to maladaptive eating habits (Peterson and Mitchell, 1999). This relapse is often triggered by re-exposure to palatable foods, food-associated cues, or stress (Herman and Polivy, 1975; Grilo et al., 1989; Drewnowski, 1997). The mechanisms underlying relapse to maladaptive eating habits in humans are unknown, and this topic has rarely been studied in animal models (Horvitz and Ettenberg, 1988; Duarte et al., 2003).

The neuronal mechanisms underlying drug reward overlap with those of food reward (Carr, 2002; DiLeone et al., 2003; Abizaid et al., 2006). Therefore, we recently adapted a reinstatement model, commonly used to study relapse to abused drugs (Shalev et al., 2002) to explore mechanisms underlying relapse to food seeking during dieting (Ghitza et al., 2006). We found that in food-restricted rats, a corticotropin-releasing factor (CRF) re-

\footnotetext{
Received Dec. 14, 2006; revised Sept. 13, 2007; accepted Sept. 13, 2007.

This work was supported by the Intramural Research Program of the National Institutes of Health-National Institute on Drug Abuse. We thank Kristina Wihbey for technical help and Dr. Rachel Batterham for helpful comments on issues related to the pharmacokinetics and duration of effects of PYY3-36.

*U.E.G. and S.G.N. contributed equally to this work.

Correspondence should be addressed to Dr. Yavin Shaham, Behavioral Neuroscience Branch, Intramural Research Program, National Institute on Drug Abuse, National Institutes of Health, 5500 Nathan Shock Drive, Baltimore, MD 21224. E-mail:yshaham@intra.nida.nih.gov.

DOI:10.1523/JNEUROSCI.5405-06.2007

Copyright $\odot 2007$ Society for Neuroscience $\quad$ 0270-6474/07/2711522-11\$15.00/0
}

ceptor antagonist blocks reinstatement of food seeking induced by yohimbine (a pharmacological stressor) but has no effect on reinstatement induced by acute noncontingent exposure to food pellets (pellet priming). These findings suggest that different mechanisms mediate relapse to food seeking induced by stress versus acute food re-exposure. Here, we explored mechanisms underlying relapse to high-fat food by studying the effect of peptide YY3-36 (PYY3-36) on reinstatement of high-fat food seeking.

PYY3-36 is a major circulatory derivative of peptide YY (PYY) (Eberlein et al., 1989), a gastrointestinal-derived hormone released from intestinal L-cells after meals in proportion to caloric intake (Tatemoto and Mutt, 1980; Murphy et al., 2006). PYY3-36 has high affinity for neuropeptide Y (NPY) Y2 receptors and lower affinity for Y1 and Y5 NPY receptors (Grandt et al., 1992; Ballantyne, 2006); NPY is a hypothalamic peptide that increases food intake after hypothalamic or ventricular injections (Leibowitz, 1995). The Y2 receptor is a putative NPY presynaptic inhibitory autoreceptor, whereas $\mathrm{Y} 1$ and $\mathrm{Y} 5$ are postsynaptic excitatory receptors (Wahlestedt et al., 1986; Larhammar and Salaneck, 2004). Systemic PYY3-36 injections decrease food intake in mice, rats, monkeys, and humans (Batterham et al., 2002, 2003; Moran et al., 2005; Chelikani et al., 2006) [but see Tschop et al. (2004) and Boggiano et al. (2005) for different results]. In rats, PYY3-36 injections into the arcuate nucleus (Arc) inhibit food intake (Bat- 
terham et al., 2002). In mice, the anorexic effects of PYY3-36 are prevented by deletion of Y2 receptors (Batterham et al., 2002; Renshaw and Batterham, 2005).

We initially assessed the effect of systemic PYY3-36 injections on ongoing high-fat pellet self-administration and on pellet priming-induced reinstatement of food seeking. We then assessed the role of $\mathrm{Y} 2$ receptors in the inhibition of pellet priminginduced reinstatement of PYY3-36 by using BIIE0246, a selective Y2 receptor antagonist (Doods et al., 1999), and the role of the Arc in this reinstatement by injecting PYY3-36 or BIIE0246 into this brain area. We then assessed the generality of PYY3-36 effects on reinstatement by examining its effect on reinstatement induced by a tone-light cue previously paired with pellet delivery (pellet cue) and the pharmacological stressor yohimbine. Finally, we assessed the specificity of PYY3-36 to reinstatement of food seeking by determining its effect on heroin priming- and heroin cue-induced reinstatement of heroin seeking.

\section{Materials and Methods}

\section{Subjects and apparatus}

Male Long-Evans rats (total $n=192,300-420$ g; Charles River, Raleigh, $\mathrm{NC}$ ) were housed in self-administration chambers for the duration of the experiment under a reverse $12 \mathrm{~h}$ light/dark cycle (lights off at 9:30 A.M.). We excluded 84 rats because of failure to meet an extinction criterion (see below), lever responding that was $>3$ SDs above the group means, poor health, catheter failure (experiment 7), or Arc cannula misplacement. The rats trained to self-administer food were kept on a restricted diet of $16 \mathrm{~g} / \mathrm{d}[\sim 60-65 \%$ of their regular daily Purina (St. Louis, MO) rat chow]. The rats trained to self-administer heroin were mildly restricted to $20-25 \mathrm{~g} / \mathrm{d}$ of Purina rat chow to maintain stable body weight. The rats' body weights were taken daily, and procedures followed the guidelines outlined in the Principles of Laboratory Animal Care (National Institutes of Health publication number 85-23). Experiments were conducted in standard self-administration chambers (Med Associates, Georgia, VT). Each chamber had two levers $9 \mathrm{~cm}$ above the floor, but only one lever ("active," retractable lever) activated the pellet dispenser, which delivered $45 \mathrm{mg}$ of chocolate-flavored food pellets containing 35\% fat and 45.2\% carbohydrate (F05879; Bioserv, San Diego, CA).

\section{Drugs}

Drugs were prepared fresh before testing. PYY3-36 (100 and $200 \mu \mathrm{g} / \mathrm{kg}$, i.p., for systemic injections and $0.4 \mu \mathrm{g}$ per side for Arc injections; catalog \#H-6042; Bachem, Torrence, CA) was dissolved in saline, BIIE0246 (2 $\mathrm{mg} / \mathrm{kg}$, i.p., for systemic injections and $0.5 \mu \mathrm{g}$ per side for Arc injections; catalog \#1700; Tocris Bioscience, Ellisville, MO) was dissolved in $30 \%$ polyethylene glycol (Sigma-Aldrich, St. Louis, MO), and yohimbine $\mathrm{HCl}$ (2 mg/kg, i.p.; Research Biochemicals, St. Louis, MO) was dissolved in distilled water. The injection volumes for systemic injections were 1 $\mathrm{ml} / \mathrm{kg}$ for PYY3-36 and BIIE0246 and $0.5 \mathrm{ml} / \mathrm{kg}$ for yohimbine. The doses of PYY3-36 and BIIE0246 for systemic and Arc injections are based on previous studies (Batterham et al., 2002; Abbott et al., 2005; Boggiano et al., 2005; Scott et al., 2005). The yohimbine dose ( $2 \mathrm{mg} / \mathrm{kg}$, i.p.) is based on our previous work (Shepard et al., 2004; Le et al., 2005; Ghitza et al., 2006). PYY3-36 was injected systemically either $30 \mathrm{~min}$ (pellet selfadministration, priming- and cue-induced reinstatement) or $60 \mathrm{~min}$ (yohimbine-induced reinstatement) before the test sessions. PYY3-36 was injected into the Arc 5 min before the test sessions. Yohimbine was injected $45 \mathrm{~min}$ before the test sessions. BIIE0246 was injected systemically 30 min before PYY3-36 injections. BIIE0246 was injected into the Arc 10 min before PYY3-36, which was injected systemically 5 min before the test sessions.

\section{Intracranial surgery and intracranial injections}

The rats were anesthetized with a mixture of sodium pentobarbital and choral hydrate (60 and $25 \mathrm{mg} / \mathrm{kg}$, i.p.). Using a stereotaxic instrument (David Kopf Instruments, Tujunga, CA), they were implanted with guide cannulas (23 gauge; Plastics One, Roanoke, VA) bilaterally 1-2 mm above the Arc: anteroposterior, $-2.3 \mathrm{~mm}$; mediolateral, $\pm 2.2 \mathrm{~mm}$; dor- soventral, -7.9 or $-8.9 \mathrm{~mm}$ ( $10^{\circ}$ angle) (Paxinos and Watson, 2005). Buprenorphine $(0.1 \mathrm{mg} / \mathrm{kg}$, s.c. $)$ was given after surgery, and the rats were allowed to recover for $7 \mathrm{~d}$. Arc PYY3-36 injections (0.4 $\mu \mathrm{g}$ per side; injection volume, $0.3 \mu \mathrm{l}$ ) and BIIE0246 injections ( $0.5 \mu \mathrm{g}$ per side; injection volume, $0.3 \mu \mathrm{l}$ ) were made with Harvard Apparatus (Holliston, MA) infusion pumps, using $10 \mu \mathrm{l}$ Hamilton (Reno, NV) syringes that were connected via polyethylene-50 tubing to 30 gauge injectors (Plastics One). The day before the intracranial injections, the rats were habituated to the injection procedure by removing and reinserting the cannula blockers. Injections were performed in the freely moving (nonanesthetized) rats over $1 \mathrm{~min}$, and injectors were left in place for an additional 2 min before being replaced with cannula blockers. After testing, the rats were perfused with paraformaldehyde (Sigma) and decapitated, and the brains were removed. Coronal sections $(40 \mu \mathrm{m})$ were sliced on a cryostat, stained with cresyl violet (ICN Biomedicals, Aurora, OH), and examined for cannula placement under a light microscope.

\section{Procedures}

With the exception of an initial assessment of the effect of PYY3-36 on ongoing food pellet self-administration, we used a reinstatement procedure that included three phases: training for food self-administration (9-12 sessions), extinction of the food-reinforced behavior (10-20 sessions), and tests for reinstatement under extinction conditions (up to 4 sessions). During all phases, the sessions started $30 \mathrm{~min}$ after the beginning of the rats' dark cycle (lights off at 9:30 A.M.). Below, we first describe the training and extinction procedures for all experiments and then provide the specific details for the reinstatement phase of each experiment. During the reinstatement phase, we counterbalanced the experimental conditions and the drug administration conditions.

\section{Food self-administration training}

All rats were given two to three $6 \mathrm{~h}$ daily sessions of "autoshaping" during which pellets were administered noncontingently every 5 min into a receptacle located near the active lever. Pellet delivery was accompanied by a compound $5 \mathrm{~s}$ tone $(2900 \mathrm{~Hz}, 20 \mathrm{~dB}$ above background)-light (a 7.5 $\mathrm{W}$ white light located above the active lever) cue; this cue is termed pellet cue. Subsequently, the rats were trained to self-administer the pellets every other day for $6 \mathrm{~h} / \mathrm{d}$ (two $3 \mathrm{~h}$ sessions separated by $1 \mathrm{~h}$ ) on a fixedratio-1 reinforcement schedule for 9-12 sessions. For the rats that were subsequently tested for food priming- and yohimbine-induced reinstatement, the timeout period after pellet delivery was $20 \mathrm{~s}$. For the rats that were subsequently tested for pellet cue-induced reinstatement, the timeout period was $40 \mathrm{~s}$.

At the start of each $3 \mathrm{~h}$ session, a red houselight was turned on, and the active lever was extended. After each pellet delivery, the pellet cue was turned on for $5 \mathrm{~s}$. At the end of each $3 \mathrm{~h}$ session, the red houselight was turned off, and the active lever was retracted. During the training days, regular food ( $16 \mathrm{~g}$ of Purina rat chow) was given immediately after the second daily session $(\sim 7.5 \mathrm{~h}$ into the dark cycle). During the off days, the $16 \mathrm{~g}$ of regular food was given at the start of the dark cycle.

We chose this training schedule and these diet conditions because previous nonoperant food-consumption studies have shown that rats placed on a restricted diet and given intermittent access to palatable food develop binge-like eating behavior (Colantuoni et al., 2002; Corwin and Buda-Levin, 2004; Avena et al., 2007) and become hypersensitive to the effect of stress on palatable food intake (Hagan et al., 2002, 2003). We used a $40 \mathrm{~s}$ timeout in the pellet cue-induced reinstatement experiment, because we found that cue-induced reinstatement of sucrose seeking is more robust with this timeout duration than with a shorter duration (J. M. Bossert, unpublished observation) (Bossert et al., 2006a).

\section{Extinction of food self-administration}

After training, the rats were given 10-20 daily extinction sessions until active lever responding was below 30 presses $/ 3 \mathrm{~h}$ for three consecutive sessions (an extinction criterion). For the rats that were subsequently tested for food priming- and yohimbine-induced reinstatement, lever presses led to tone-light cue presentations but not pellet delivery. For the rats that were subsequently tested for pellet cue-induced reinstatement, lever presses had no programmed consequences (i.e., neither the tonelight cue nor pellets were made available). Initially, the rats were given 
two $3 \mathrm{~h}$ sessions (separated by $1 \mathrm{~h}$ ) each day for $6 \mathrm{~d}$. Subsequently, they were given one $3 \mathrm{~h}$ extinction session per day for an additional 4-14 d until they met the extinction criterion. During the extinction and reinstatement phases, the regular food ( $16 \mathrm{~g}$ ) was given approximately at the same time as during training (i.e., $\sim 7.5 \mathrm{~h}$ after the onset of the dark cycle).

\section{Experiment 1: effect of systemic PYY3-36 injections on} food self-administration

In this initial experiment, we examined the effect of systemic PYY3-36 injections on ongoing food self-administration. After 12 training days, we assessed the effect of PYY3-36 on food pellet self-administration in two $6 \mathrm{~h}$ tests (two $3 \mathrm{~h}$ sessions separated by $1 \mathrm{~h}$ ) that were conducted $48 \mathrm{~h}$ apart. We used the within-subjects factors of PYY3-36 pretreatment (vehicle, PYY3-36) and session (first $3 \mathrm{~h}$ session, second $3 \mathrm{~h}$ session) and the between-subject factor of PYY3-36 dose (100 and $200 \mu \mathrm{g} / \mathrm{kg} ; n=8-10$ per dose). Thus, each rat was given an injection of the PYY3-36 vehicle (saline) and a single PYY3-36 dose before the test sessions, which were performed in a counterbalanced order.

\section{Experiment 2: effect of systemic PYY3-36 injections on pellet} priming-induced reinstatement

After testing the effect of PYY3-36 on food self-administration (experiment 1 ), the same rats were given extinction sessions (see above) and were subsequently tested for pellet priming-induced reinstatement in $3 \mathrm{~h}$ test sessions in which one food pellet was administered noncontingently just before the start of the test sessions. We tested the effect of PYY3-36 on pellet priming-induced reinstatement in four $3 \mathrm{~h}$ test sessions with two sessions run consecutively and 1 extinction day between sets of tests. We used the within-subjects factors of PYY3-36 pretreatment (vehicle, PYY3-36), pellet priming (pellet, no pellet), and session minutes (six 30 min blocks) and the between-subject factor of PYY3-36 dose (100 and $200 \mu \mathrm{g} / \mathrm{kg} ; n=8-10$ per dose). Thus, each rat in the low PYY3-36 dose group was given four counterbalanced test sessions [vehicle-no pellet, vehicle-pellet, PYY3-36 (100 $\mu \mathrm{g} / \mathrm{kg})$-no pellet, and PYY3-36 (100 $\mu \mathrm{g} /$ $\mathrm{kg}$ )-pellet], and each rat in the high-dose group was given four counterbalanced test sessions [vehicle-no pellet, vehicle-pellet, PYY3-36 (200 $\mu \mathrm{g} / \mathrm{kg})$-no pellet, and PYY3-36 (200 $\mu \mathrm{g} / \mathrm{kg})$-pellet]. We used a similar experimental design, which is based on previous pharmacological studies using the reinstatement model (Shaham et al., 1997), in the other experiments described below. The rationale for using this mixed experimental design is to decrease the number of rats used while limiting the number of repeated pellet priming-induced reinstatement tests to two (instead of four in the case of a complete within-subjects design) to minimize habituation to the effect of pellet priming (or other stimuli used to induce reinstatement) over repeated testing.

A separate group of rats $(n=10)$ was tested in the same manner as the rats that received $200 \mu \mathrm{g} / \mathrm{kg}$ PYY3-36 (see above), with the exception that this group of rats received an injection of the Y2 receptor antagonist BIIE0246 (2 mg/kg, i.p.) $30 \mathrm{~min}$ before injections of PYY3-36 or its vehicle.

\section{Experiment 3: effect of Arc PYY3-36 injections on pellet} priming-induced reinstatement

The purpose of experiment 3 was to determine whether the systemic effect of PYY3-36 on pellet priming-induced reinstatement is mimicked by Arc injections of the peptide. We tested the effect of Arc injections of PYY3-36 (0.4 $\mu \mathrm{g}$ per side) on pellet priming-induced reinstatement in four test sessions with two sessions run consecutively and 1 extinction day between each set of tests $(n=14)$. For this purpose, we used the within-subjects factors of PYY3-36 pretreatment (vehicle, $0.4 \mu \mathrm{g}$ per side), pellet priming (pellet, no pellet), and session minutes.

\section{Experiment 4: effect of Arc BIIE0246 injections on the inhibitory} effect of systemic injections of PYY3-36 on pellet priming-induced reinstatement

Based on the results of experiments 2 and 3 , in experiment 4 we explored the role of Arc Y2 receptors in the inhibitory effect of systemic injections of PYY3-36 on pellet priming-induced reinstatement. The rats $(n=10)$ received Arc injections of BIIE0246 (0.5 $\mu$ g per side) or its vehicle $10 \mathrm{~min}$ before systemic injections of PYY3-36 (200 $\mu \mathrm{g} / \mathrm{kg}$, i.p.); PYY3-36 was injected $5 \mathrm{~min}$ before the test sessions. We tested the rats in four test sessions with two sessions run consecutively and 1 extinction day between each set of tests, using the within-subjects factors of BIIE0246 dose ( 0 and $0.5 \mu$ g per side) and pellet priming (pellet, no pellet). The order of the experimental conditions was counterbalanced.

\section{Experiment 5: effect of systemic and Arc PYY3-36 injections on cue-induced reinstatement}

The purpose of experiment 5 was to assess the generality of the effect of PYY3-36 on reinstatement by determining the effect of the drug on reinstatement of food seeking induced by discrete cues previously associated with pellet delivery during training. It is well established that such discrete cues robustly reinstate both food and drug seeking after extinction (De Vries and Schoffelmeer, 2005; See, 2005). As stated previously, during the training phase each pellet delivery was paired with a tone-light cue (pellet cue); this pellet cue was not presented during the extinction phase after lever pressing. During the tests for reinstatement, lever responding led to contingent presentations of the tone-light cue under the fixed-ratio-1 40 s timeout reinforcement schedule. We tested the effect of PYY3-36 on cue-induced reinstatement in four test sessions with two sessions run consecutively and 5 extinction days between sets of tests. We conducted 5 extinction days between sets of tests, because in previous studies we found that this procedure minimizes habituation to the effect of the conditioned cues on reinstatement of sucrose seeking (J. M. Bossert, unpublished observation) (Bossert et al., 2006). We used the within-subjects factors of PYY3-36 pretreatment (vehicle, PYY3-36), pellet cue (cue, no cue), and session minutes and the between-subject factor of PYY3-36 dose (100 and $200 \mu \mathrm{g} / \mathrm{kg} ; n=10$ per dose).

Based on the results of experiment 3, we also assessed the effect of Arc PYY3-36 injections on pellet cue-induced reinstatement of food seeking $(n=8)$. The experimental procedure for self-administration training and extinction of lever responding was the same as described for the rats given systemic PYY3-36 injections. Rats were given injections of PYY3-36 (0.4 $\mu \mathrm{g}$ per side) or its vehicle in four test sessions with two sessions run consecutively and 5 extinction days between sets of tests. We used the within-subjects factors of PYY3-36 pretreatment (vehicle, 0.4 $\mu \mathrm{g}$ per side), pellet cue (cue, no cue), and session minutes.

\section{Experiment 6: effect of systemic PYY3-36 injections on} yohimbine-induced reinstatement

The purpose of experiment 6 was to further assess the generality of the effect of PYY3-36 on reinstatement by determining its effect on reinstatement induced by yohimbine, an $\alpha-2$ adrenoceptor antagonist that induces stress- and anxiety-like responses in both humans and nonhumans (Bremner et al., 1996a,b) and reinstates drug and food seeking in laboratory animals (Lee et al., 2004; Bossert et al., 2005; Le et al., 2005; Nair et al., 2006). We tested the effect of PYY3-36 on yohimbine-induced reinstatement in four test sessions with two sessions run consecutively and 1 extinction day between sets of tests. We used the within-subjects factors of PYY3-36 pretreatment (vehicle, PYY3-36), yohimbine (vehicle, $2 \mathrm{mg} /$ $\mathrm{kg}$ ), and session minutes and the between-subject factor of PYY3-36 dose (100 and $200 \mu \mathrm{g} / \mathrm{kg} ; n=9-10$ per dose). One outlier rat that lever pressed $>350$ times in both the vehicle and the PYY3-36 (100 $\mu \mathrm{g} / \mathrm{kg}$, i.p. $)$ condition ( $>3 \mathrm{SD}$ above the group mean) was excluded.

Experiment 7: effect of systemic PYY3-36 injections on heroin cueand heroin priming-induced reinstatement of heroin seeking In this final experiment, we assessed the specificity of PYY3-36 effect to reinstatement of food seeking by determining its effect on heroin priming- and heroin cue-induced reinstatement of heroin seeking. Rats were implanted with intravenous catheters into the jugular vein as described previously (Shaham et al., 1996; Shalev et al., 2001a; Bossert et al., 2006b), and training for heroin self-administration started after 7 recovery days. During this time period and the training phase, catheters were flushed every $24-48 \mathrm{~h}$ with gentamicin in sterile saline $(0.08 \mathrm{mg} / \mathrm{ml})$. Experiment 7 consisted of three phases: training for heroin selfadministration, extinction of the heroin-reinforced behavior, and tests for the effect of PYY3-36 on heroin cue-induced or heroin priming- 
induced reinstatement of heroin seeking. All sessions started $30 \mathrm{~min}$ after the onset of the dark cycle.

The rats were trained to self-administer heroin for $6 \mathrm{~h} / \mathrm{d}$ for $10 \mathrm{~d}$ under a fixed-ratio-1 schedule. Heroin (diacetylmorphine $\mathrm{HCl}$; National Institute on Drug Abuse) was dissolved in sterile saline and infused in a volume of $65 \mu$ lover $2.3 \mathrm{~s}$ at a dose of $0.05 \mathrm{mg} / \mathrm{kg}$ (first five sessions) and $0.025 \mathrm{mg} / \mathrm{kg}$ (last five sessions) per infusion. The heroin training doses are based on previous studies (Shalev et al., 2001b; Bossert et al., 2004), and the unit dose was halved after the fifth day of training to verify that the rats reliably acquired drug self-administration, as indicated by the increase in lever presses for the lower dose (Yokel, 1987). During training, heroin infusions were earned under a fixed-ratio-1 (40 s timeout) schedule and were accompanied by the compound tone-light cue for $5 \mathrm{~s}$. Sessions began with the illumination of the red houselight and the insertion of the active lever. At the end of each session, the houselight was turned off, the active lever was retracted, and the rats were fed $20-25 \mathrm{~g}$ to maintain their body weight. During the extinction phase $(10 \mathrm{~d})$, procedures were identical to those of training, except that the responses on the active lever did not result in the infusion of heroin or the presentation of the tone-light cue.

Heroin cue-induced reinstatement. We tested the effect of PYY3-36 on heroin cue-induced reinstatement in four test sessions with two sessions run consecutively and 5 extinction days between sets of tests. In the four counterbalanced test sessions, the rats were given injections of vehicle (saline) or PYY3-36 (100 or $200 \mu \mathrm{g} / \mathrm{kg}$, i.p.) $30 \mathrm{~min}$ before the start of the tests, and active lever presses led to the presentation of either the tonelight cue or no cue. We used the within-subjects factors of PYY3-36 pretreatment (vehicle, PYY3-36), heroin cue (cue, no cue), and session minutes and the between-subject factor of PYY3-36 dose (100 and 200 $\mu \mathrm{g} / \mathrm{kg} ; n=8-10$ per dose).

Heroin priming-induced reinstatement. At the completion of the heroin cue-induced reinstatement tests, nine rats were given four additional extinction sessions in the presence of the tone-light cue. During two subsequent test sessions, four of these rats were given injections of the PYY3-36 vehicle (saline, intraperitoneally) $30 \mathrm{~min}$ before the sessions, and $25 \mathrm{~min}$ later they were given injections of heroin $(0.25 \mathrm{mg} / \mathrm{kg}$, s.c.) or its vehicle (saline, subcutaneously). The other five rats were given injections of $200 \mu \mathrm{g} / \mathrm{kg}$ PYY3-36 and heroin or saline before the test sessions. Thus, we used the within-subjects factor of heroin priming ( 0 and 0.25 $\mathrm{mg} / \mathrm{kg}$ ) and the between-subjects factor of PYY3-36 dose (0 and 200 $\mu \mathrm{g} / \mathrm{kg}$ ). The heroin priming dose is based on previous studies (Shaham et al., 1996; Shalev et al., 2001b).

\section{Statistical analyses}

The data on the effect of PYY3-36 on food pellet self-administration (experiment 1) were analyzed separately for the number of pellets earned and timeout nonreinforced active lever responding. The data from the reinstatement experiments were analyzed for nonreinforced lever presses on the previously active lever and on the inactive lever. Because the experimental manipulations had no effect on inactive lever responding, which was very low, these data are not shown.

In reinstatement experiments in which two PYY3-36 doses were used, we tested the effect of PYY3-36 in a factorial design with the withinsubjects factors of PYY3-36 pretreatment (vehicle, PYY3-36), stimulus condition [pellet priming (pellet, no pellet), pellet cue/heroin cue (cue, no cue), or yohimbine (vehicle, $2 \mathrm{mg} / \mathrm{kg}$ )], and session minutes (six 30 min blocks) and the between-subject factor of PYY3-36 dose (100 and $200 \mu \mathrm{g} / \mathrm{kg}$ ). In this analysis, a significant effect of PYY3-36 pretreatment reflects differences between PYY3-36 (100 or $200 \mu \mathrm{g} / \mathrm{kg}$, or both) and vehicle, whereas a significant effect of PYY3-36 dose reflects differences between the $100 \mu \mathrm{g} / \mathrm{kg}$ dose and the $200 \mu \mathrm{g} / \mathrm{kg}$ dose. The factors used in the statistical analysis of reinstatement of lever responding in which only one PYY3-36 dose was used are described in Results. Because of the complexity of the experimental design used, we primarily report in Results critical significant interactions between the different factors. These significant interactions were followed by post hoc PLSD tests (two-tailed), which are indicated in the figures.

\section{Results}

During the food training phase, the rats (total $n=108$ ) were maintained on $16 \mathrm{~g} / \mathrm{d}$ of regular chow food and were given $6 \mathrm{~h}$ access to the food pellets every other day. They gained weight when pellets were available and lost weight when they were not (Fig. $1 A$ ). A repeated-measures ANOVA using pellet availability and training day as the within-subjects factors and body weight as the dependent measure revealed a significant interaction between these factors $(p<0.01)$. This weight fluctuation was not observed during the extinction and reinstatement phases when the pellets were not available.

\section{Training and extinction}

The rats in experiment 1-6 were trained for 9-12 sessions and demonstrated reliable pellet self-administration and, as in our previous study, a progressive escalation of timeout responding across sessions (Ghitza et al., 2006) (Fig. 1). The rats were then given six $6 \mathrm{~h}$ extinction sessions and additional $3 \mathrm{~h}$ extinction sessions during which lever presses decreased over time (Fig. 1). The rats that were subsequently tested for pellet priming- and yohimbine-induced reinstatement $(n=80)$ (Fig. $1 A-C)$ were trained under a fixed-ratio-1 $20 \mathrm{~s}$ timeout reinforcement schedule, and their active lever presses were extinguished in the presence of the pellet cue (the $5 \mathrm{~s}$ tone-light previously paired with each pellet delivery). The rats that were subsequently tested for pellet cue-induced reinstatement $(n=28)$ (Fig. $1 A, D, E)$ were trained under a fixed-ratio-1 $40 \mathrm{~s}$ timeout reinforcement schedule, and their active lever presses were extinguished in the absence of the pellet cue.

During the training phase for both experimental conditions, the statistical analyses revealed significant increases over time for both pellets earned and active-lever timeout responding ( $p$ values $<0.01$ ) but not for inactive lever responding. During the extinction phase for both experimental conditions, the analyses revealed significant decreases over time for active lever responding during the first 6 extinction days when the rats were given two $3 \mathrm{~h}$ daily sessions that were separated by $1 \mathrm{~h}$ ( $p$ values $<0.01)$ but not for inactive lever responding.

\section{Experiment 1: effect of systemic PYY3-36 injections on food self-administration}

PYY3-36 modestly decreased the number of pellets earned (Fig. 2) and timeout lever responding (data not shown) during food self-administration; however, this effect did not reach statistical significance and was not dose dependent. Two groups of rats $(n=$ 8-10 per group) were given injections of one dose of PYY3-36 ( 100 or $200 \mu \mathrm{g} / \mathrm{kg}$ ) or vehicle $30 \mathrm{~min}$ before 2 training days during which the rats lever pressed for the pellets in two $3 \mathrm{~h}$ sessions that were separated by $1 \mathrm{~h}$. The statistical analyses for each measure (pellet and timeout responses) included the within-subjects factors of PYY3-36 pretreatment (vehicle, PYY3-36) and session (first $3 \mathrm{~h}$ session, second $3 \mathrm{~h}$ session) and the between-subject factor of PYY3-36 dose (100 and $200 \mu \mathrm{g} / \mathrm{kg}$ ). The analysis for the effect of PYY3-36 on the number of pellets earned revealed an approaching significant effect of PYY3-36 pretreatment $\left(F_{(1,16)}=\right.$ $4.1 ; p=0.061)$; the effects of PYY3-36 dose or session or the interactions between the different factors were not significant $(p>0.05)$. The analysis for the effect of PYY3-36 on the number of timeout responses did not reveal any significant effects, with the exception of session $\left(F_{(1,16)}=6.3 ; p<0.05\right)$, because of a greater number of timeout responses in the first session than in the second session, collapsed across PYY3-36 pretreatment conditions (data not shown). 

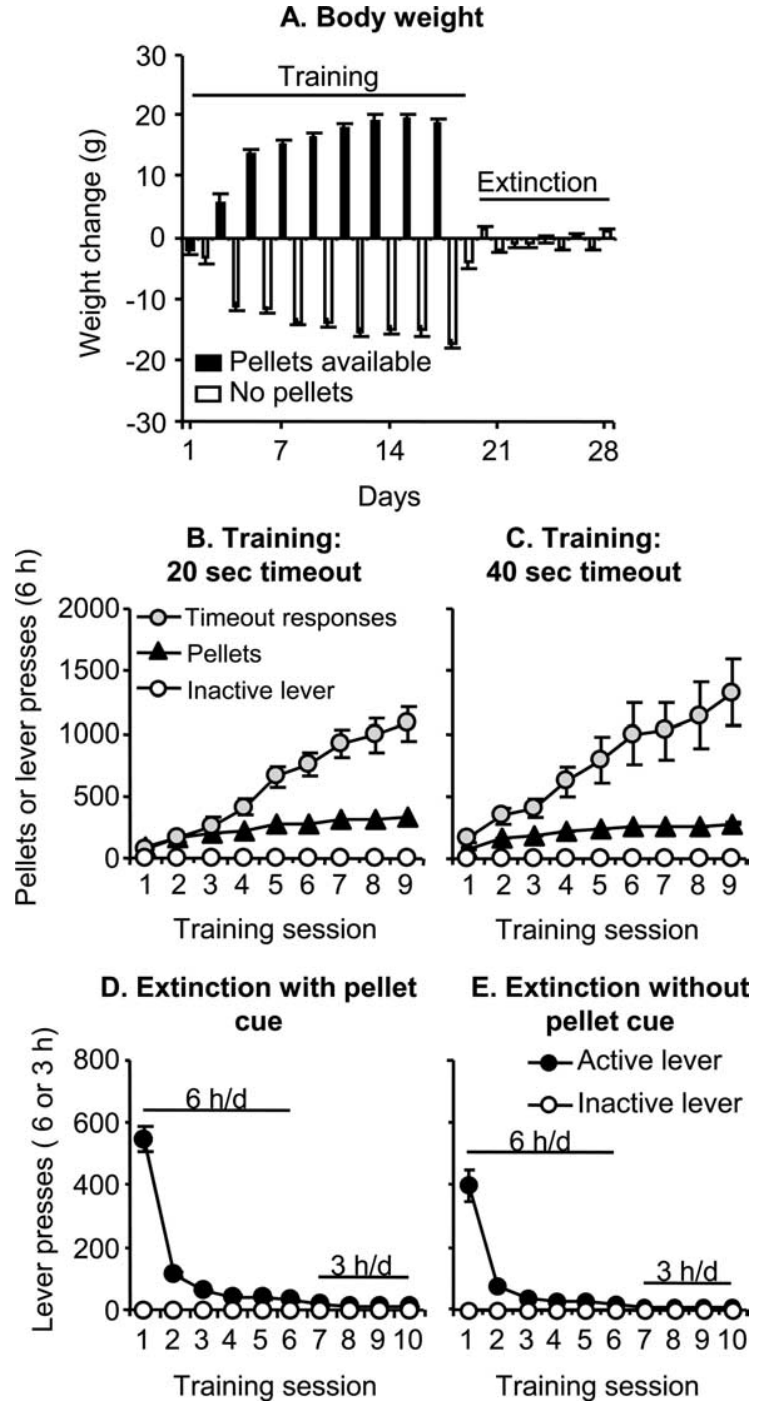

Figure 1. Food pellet self-administration training and extinction of the food-reinforced lever responding. $\boldsymbol{A}$, Body weight fluctuations during the training and extinction phases. During the training phase, the rats (total $n=108$ ) were maintained on $16 \mathrm{~g} / \mathrm{d}$ of regular chow food and were given $6 \mathrm{~h}$ access to the food pellets every other day. During the extinction (and reinstatement) phases, food pellets were not available in the selfadministration chambers, and the rats were maintained on $16 \mathrm{~g} / \mathrm{d}$ of regular food. $\boldsymbol{B}$ Training. Mean \pm SEM number of $35 \%$ fat pellets earned, active lever presses, and inactive lever presses during the training sessions over 9 alternating days (two $3 \mathrm{~h}$ sessions per day, every other day) for rats that were trained under a fixed-ratio-1 (FR-1) $20 \mathrm{~s}$ timeout reinforcement schedule $(n=80)$; these rats were subsequently tested for pellet primingand yohimbine-induced reinstatement. $C$, Corresponding training data for rats that were trained under an FR-1 $40 \mathrm{~s}$ timeout reinforcement schedule $(n=28)$; these rats were subsequently tested for pellet cue-induced reinstatement. $\boldsymbol{D}$, Extinction with pellet cue. Mean number of presses on the previously active lever or the inactive lever during the extinction phase for rats that had been trained with a timeout period of $20 \mathrm{~s}$ after each pellet delivery. Lever presses were extinguished in the presence of the tone-light pellet cue over consecutive days for two $3 \mathrm{~h}$ sessions per day on days $1-6$ and for $3 \mathrm{~h} / \mathrm{d}$ for the subsequent days. $\boldsymbol{E}$, Extinction without pellet cue. Corresponding extinction data for rats that had been trained with a timeout period of $40 \mathrm{~s}$ after each pellet delivery and lever presses extinguished in the absence of the pellet cue are shown.

Experiment 2: effect of systemic PYY3-36 injections on pellet priming-induced reinstatement

Systemic injections of both doses of PYY3-36 decreased pellet priming-induced reinstatement of active lever presses, an effect that was most pronounced in the first $30 \mathrm{~min}$ of the $3 \mathrm{~h}$ test sessions (Fig. $3 A, B$ ). The ANOVA revealed significant interac-
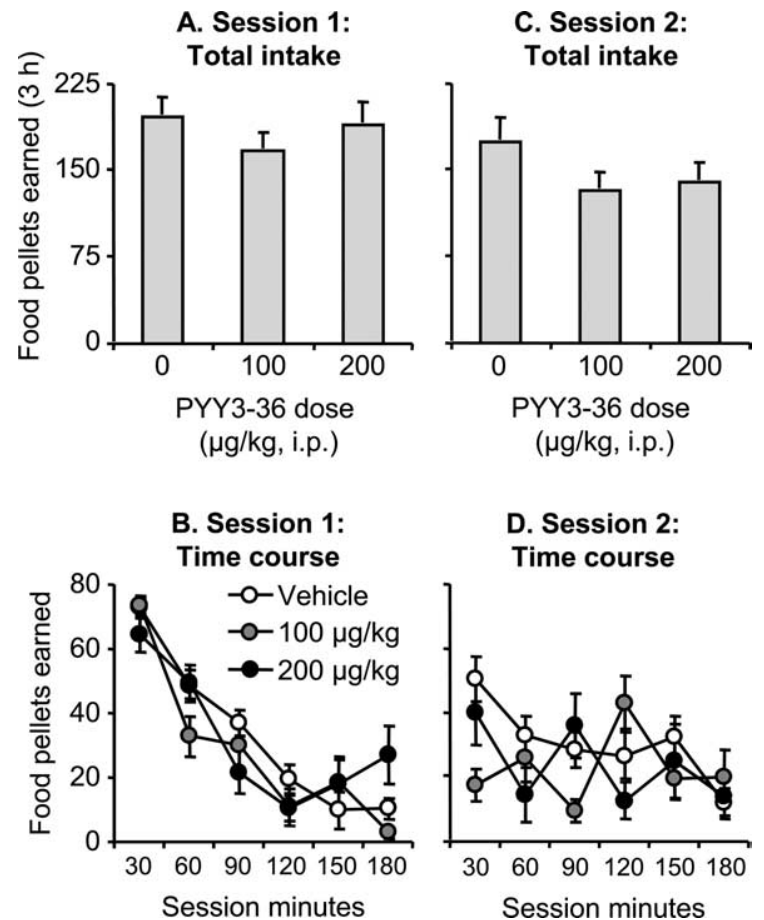

Figure 2. Systemic PYY3-36 injections have a minimal effect on pellet self-administration. $\boldsymbol{A}, \boldsymbol{C}$, Total pellets. Mean \pm SEM number of $35 \%$ fat pellets self-administered after vehicle (saline) or PYY3-36 injections during the first $(\boldsymbol{A})$ and second $(\boldsymbol{C}) 3 \mathrm{~h}$ sessions $(n=8-10$ for each PYY3-36 dose; $n=18$ for the vehicle condition). $\boldsymbol{B}, \boldsymbol{D}$, Corresponding time course for the data described in $\boldsymbol{A}$ and $\boldsymbol{C}$. PYY3-36 or vehicle was injected 30 min before session 1; session 2 started $1 \mathrm{~h}$ after the end of session 1. Each rat was given injections of vehicle and one dose of PYY3-36 (see Materials and Methods and Results for details of the experimental design).

tion effects of PYY3-36 pretreatment $\times$ pellet priming $\left(F_{(1,16)}=\right.$ 32.5; $p<0.01)$ and PYY3-36 pretreatment $\times$ pellet priming $X$ session minutes $\left(F_{(5,80)}=48.3 ; p<0.01\right)$. Injections of the $\mathrm{Y} 2$ receptor antagonist BIIE0246 (2 $\mathrm{mg} / \mathrm{kg}$, i.p.) $30 \mathrm{~min}$ before PYY3-36 injections reversed the inhibition of PYY3-36 on pellet priming-induced reinstatement (Fig. 3C,D); injections of BIIE0246 by itself had no effect on pellet priming-induced reinstatement. As described previously, for determining the effect of BIIE0246 on the inhibition of PYY3-36 on pellet priminginduced reinstatement, we pretreated one group of rats during four test sessions with the Y2 antagonist. These rats were given injections of $200 \mu \mathrm{g} / \mathrm{kg}$ PYY3-36 or its vehicle and tested in extinction (no pellet) or after a single exposure to a food pellet (pellet priming). The statistical analysis for this group included the within-subjects factors of PYY3-36 pretreatment (vehicle, 200 $\mu \mathrm{g} / \mathrm{kg}$ ), pellet priming (pellet, no pellet), and session minutes. This analysis revealed significant effects for pellet priming $\left(F_{(1,9)}\right.$ $=59.5 ; p<0.01)$ and session minutes $\left(F_{(5,45)}=58.0 ; p<0.01\right)$ but no significant effects of PYY3-36 pretreatment or interactions between the different factors $(p>0.05)$. These results indicate that in the presence of BIIE0246, systemic injections of PYY3-36 did not attenuate pellet priming-induced reinstatement.

\section{Experiment 3: effect of Arc PYY3-36 injections on pellet} priming-induced reinstatement

Arc injections of PYY3-36 decreased pellet priming-induced reinstatement (Fig. $4 A, B$ ), an effect that was most pronounced in the first $30 \mathrm{~min}$ of the $3 \mathrm{~h}$ test sessions. The statistical analysis included the within-subjects factors of PYY3-36 pretreatment (vehicle, $0.4 \mu \mathrm{g}$ per side), pellet priming (pellet, no pellet), and 
A. Total presses

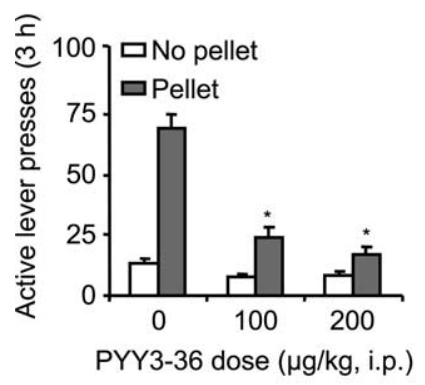

B. Time course

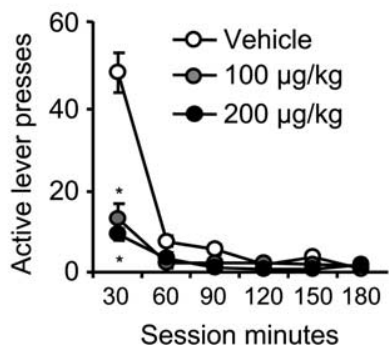

C. Total presses Y2 antagonist

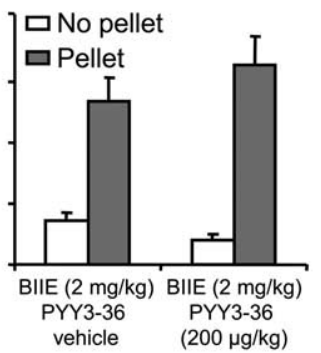

D. Time course Y2 antagonist

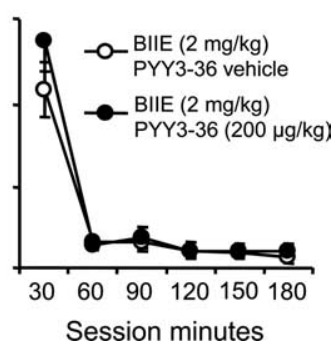

Figure 3. Systemic PYY3-36 injections attenuate pellet priming-induced reinstatement of food seeking, an effect reversed by systemic injections of the Y2 receptor antagonist BIIE0246 (BIIE). $A$, Mean \pm SEM number of nonreinforced active lever presses in the no pellet (extinction) and the pellet-priming conditions after vehicle or PYY3-36 injections ( $n=8-10$ for each PYY3-36 dose; $n=18$ for the vehicle condition). PYY3-36 or vehicle was injected 30 min before the test sessions. $\boldsymbol{B}$, Time course of the data described in $\boldsymbol{A}$. $\boldsymbol{C}$, Mean number of active lever presses after BIIE0246 pretreatment ( $30 \mathrm{~min}$ ) and subsequent injections of PYY3-36 or its vehicle $(n=10)$. $\boldsymbol{D}$, Time course of the data described in $C^{*} p<0.05$, different from vehicle-pellet condition.

session minutes. The ANOVA revealed significant interaction effects of PYY3-36 pretreatment $\times$ pellet priming $\left(F_{(1,13)}=15.8\right.$; $p<0.01)$ and PYY3-36 pretreatment $\times$ pellet priming $X$ session minutes $\left(F_{(5,65)}=7.5 ; p<0.01\right)$. PYY3-36 had no effect on pellet priming-induced reinstatement in 14 rats with cannulas bilaterally in the ventromedial hypothalamus (VMH), or with one cannula in the Arc and the second cannula in the VMH. The mean \pm SEM active lever presses per $3 \mathrm{~h}$ were $69 \pm 7$ and $74 \pm 16$ for the vehicle and PYY3-36 conditions, respectively.

Experiment 4: effect of Arc BIIE0246 injections on the inhibitory effect of systemic injections of PYY3-36 on pellet priming-induced reinstatement

Arc injections of BIIE0246 had no effect on inhibition of pellet priming-induced reinstatement by PYY3-36 (Fig. 4C,D). In this experiment, all rats were given Arc injections of BIIE0246 or its vehicle and were then given injections systemically with PYY3-36 $(200 \mu \mathrm{g} / \mathrm{kg})$ before the test session. The statistical analysis included the within-subjects factor of BIIE0246 dose (0, $0.5 \mu \mathrm{g}$ per side) and pellet priming (pellet, no pellet). The ANOVA revealed no significant effects of BIIE0246 dose, pellet priming, or interactions between the two factors ( $p$ values $>0.1$ ).

Experiment 5: effect of systemic and Arc injections of PYY336 injections on cue-induced reinstatement

Systemic injections of both doses of PYY3-36 decreased pellet cue-induced reinstatement of active lever presses, an effect that was most pronounced in the first $30 \mathrm{~min}$ of the $3 \mathrm{~h}$ test sessions
(Fig. 5A, $B$ ). The ANOVA revealed significant interaction effects of PYY3-36 pretreatment $\times$ pellet cue $\left(F_{(1,18)}=24.9 ; p<0.01\right)$ and PYY3-36 pretreatment $\times$ pellet cue $X$ session minutes $\left(F_{(5,90)}=17.4 ; p<0.01\right)$. In contrast, Arc injections of PYY3-36 did not decrease pellet cue-induced reinstatement of food seeking; surprisingly, these injections appeared to enhance the response to the discrete cue and to induce reinstatement of lever responding in the absence of this cue. The statistical analysis included the within-subjects factors of PYY3-36 pretreatment (vehicle, $0.4 \mu \mathrm{g}$ per side), pellet cue (cue, no cue), and session minutes. The ANOVA revealed significant effect of pellet cue $\left(F_{(1,7)}=17.8 ; p<0.01\right)$ and PYY3-36 pretreatment $\left(F_{(1,7)}=6.7\right.$; $p<0.05)$ but no significant interaction between PYY3-36 pretreatment $\times$ pellet cue $\times$ session minutes $(p>0.05)$. The significant effect of PYY3-36 pretreatment reflects enhanced nonreinforced lever presses by Arc PYY3-36 injections after extinction, regardless of discrete cue exposure.

\section{Experiment 6: effect of systemic PYY3-36 injections on yohimbine-induced reinstatement}

Systemic PYY3-36 injections had no effect on yohimbineinduced reinstatement (Fig. 6). The ANOVA only revealed a significant effect of yohimbine $\left(F_{(1,17)}=42.8 ; p<0.01\right)$ but no interaction effects between the different factors ( $p$ values $>0.05$ ).

\section{Experiment 7: effect of systemic PYY3-36 injections on} cue-induced and heroin priming-induced reinstatement of heroin seeking

The rats in experiment 7 were trained for 10 sessions and demonstrated reliable heroin self-administration and, interestingly, as in the food-trained rats, also demonstrated a progressive escalation of timeout responding across sessions (Fig. 7). The rats were then given six $6 \mathrm{~h}$ extinction sessions and additional $3 \mathrm{~h}$ extinction sessions during which lever presses decreased over time (Fig. 7). Systemic injections of both doses of PYY3-36 had no effect on heroin cue-induced or heroin priming-induced reinstatement of heroin seeking. For heroin cue-induced reinstatement, the ANOVA revealed a significant effect of heroin cue $\left(F_{(1,14)}=21.8 ; p<0.01\right)$, whereas the effects of PYY3-36 pretreatment or PYY3-36 pretreatment $X$ heroin cue were not significant ( $p$ values $>0.05$ ) (Fig. $7 C$ ). For heroin priming-induced reinstatement, the ANOVA revealed a significant effect of heroin priming $\left(F_{(1,7)}=6.4 ; p<0.05\right)$, whereas the effects of PYY3-36 pretreatment or PYY3-36 pretreatment $X$ heroin priming were not significant ( $p$ values $>0.05$ ) (Fig. $7 D)$.

\section{Discussion}

We used a reinstatement model to explore PYY3-36 effects on relapse to food seeking in food-restricted rats. We found that systemic PYY3-36 injections attenuated pellet priming- and pellet cue-induced reinstatement of food seeking. In contrast, systemic PYY3-36 injections had minimal effects on ongoing food self-administration, reinstatement of food seeking induced by the pharmacological stressor yohimbine, or heroin priming- or heroin cue-induced reinstatement. We also found that Arc PYY3-36 injections decreased pellet priming- but not cue-induced reinstatement and that the systemic effect of PYY3-36 on pellet priming-induced reinstatement was reversed by systemic but not Arc injections of the Y2 receptor antagonist BIIE0246. These data indicate that PYY3-36 systemic effects on reinstatement of food seeking are likely independent of Y2 receptor activation in Arc. 


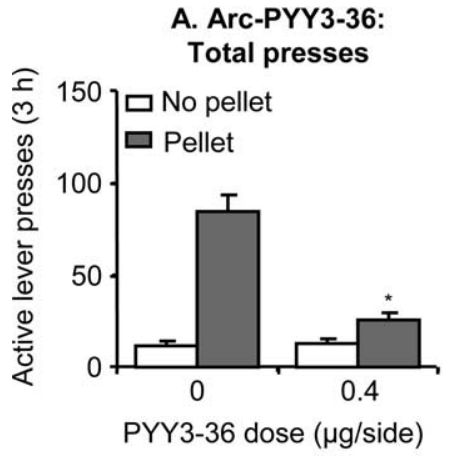

B. Arc-PYY3-36: Time course

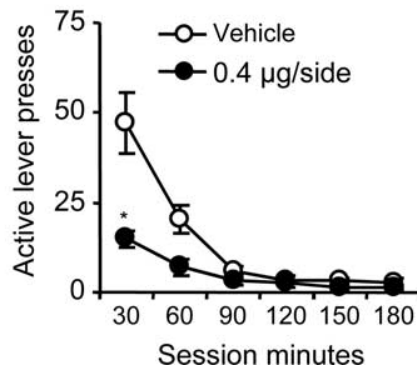

C. Systemic PYY3-36-ArcBIIE: Total presses

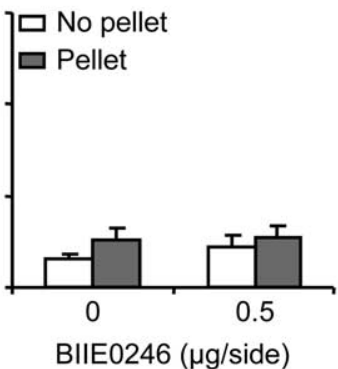

D. Systemic PYY3-36Arc-BIIE: Time course

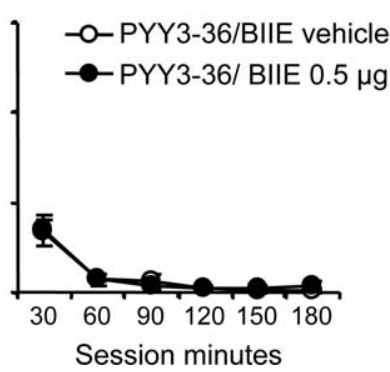

\section{E. Cannulae placement}

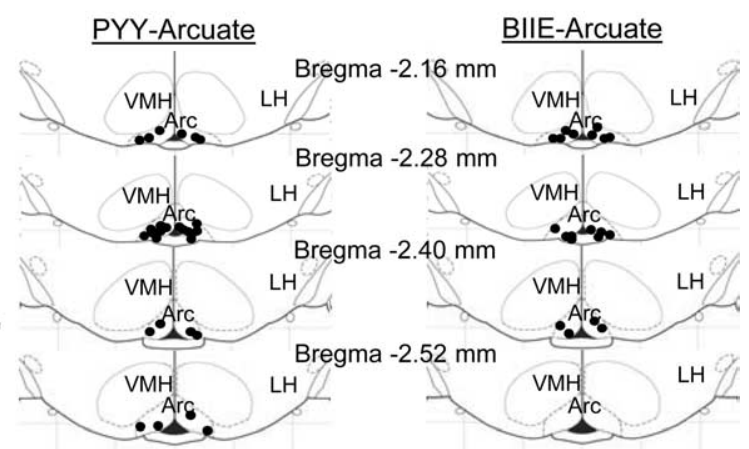

Figure 4. Arc PYY3-36 injections attenuate pellet priming-induced reinstatement, whereas Arc injections of BIIE0246 (BIIE) do not block the inhibition of pellet priming-induced reinstatement by systemic injections of PYY3-36. A, Mean \pm SEM number of active lever presses in the pellet-priming and no-pellet conditions. PYY3-36 or vehicle was injected 5 min before the test sessions $(n=$ 14). ${ }^{*} p<0.05$, different from vehicle-pellet condition. $\boldsymbol{B}$, Time course of the data described in $\boldsymbol{A}$. $\boldsymbol{C}$, Mean number of active lever presses in the pellet-priming and no-pellet conditions in rats that received Arc injections of BIIE0246 or its vehicle and, 10 min later, systemic injections of PYY3-36 $(n=10)$. D, Time course of the data described in $\boldsymbol{C}$. $\boldsymbol{E}$, Approximate placements of the tip of the injector (Paxinos and Watson, 2005). LH, Lateral hypothalamus.
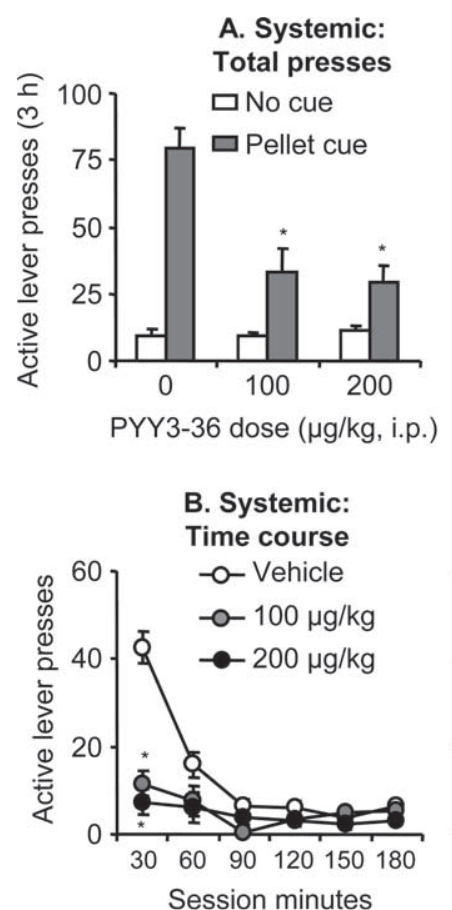

C. Arcuate:

Total presses

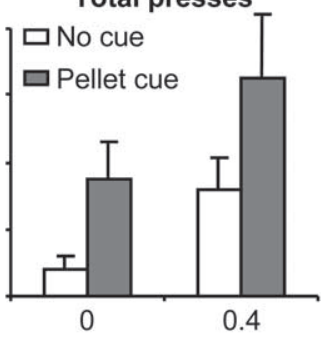

PYY3-36 dose $(\mu \mathrm{g} / \mathrm{side})$

D. Arcuate:

Time course

-0 -Vehicle

$\rightarrow 0.4 \mu \mathrm{g} / \mathrm{side}$

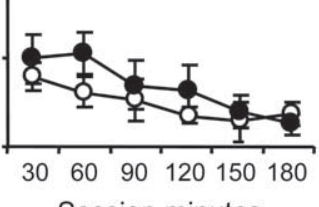

\section{E. Cannulae placement}

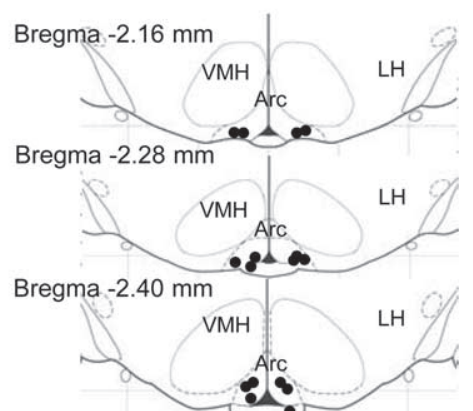

Bregma $-2.52 \mathrm{~mm}$

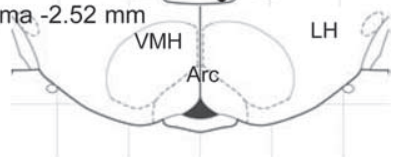

Figure 5. Systemic but not Arc PYY3-36 injections attenuate pellet cue-induced reinstatement of food seeking. $A$, Mean $\pm S E M$ number of active lever presses in the no-cue (extinction) and cue conditions after systemic injections of vehicle or PYY3-36 injections ( $n=10$ for each PYY3-36 dose; $n=20$ for the vehicle condition). $\boldsymbol{B}$, Time course of the data described in $\boldsymbol{A}$. $\boldsymbol{C}$, Mean number of active lever presses in the pellet-cue and no-cue conditions in rats that received Arc injections of PYY3-36 or its vehicle $(n=8)$. $\boldsymbol{D}$, Time course of the data described in $\boldsymbol{C}$. $\boldsymbol{E}$, Approximate placements of the tip of the injectors of the rats. ${ }^{*} p<0.05$, different from vehicle-cue condition. LH, Lateral hypothalamus. 

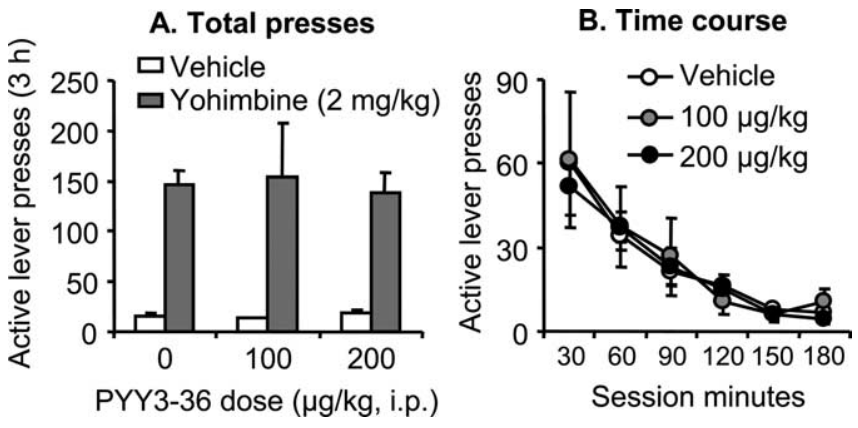

Figure 6. Systemic PYY3-36 injections have no effect on yohimbine-induced reinstatement of food seeking. $A$, Mean \pm SEM number of active lever presses after injections of yohimbine or its vehicle (distilled water) in rats pretreated with PYY3-36 or its vehicle ( $n=9-10$ for each PYY3-36 dose; $n=19$ for the vehicle condition). PYY3-36 or its vehicle was injected 15 min before yohimbine or its vehicle. Yohimbine was injected $45 \mathrm{~min}$ before the session. $\boldsymbol{B}$, Time course of the data described in $\boldsymbol{A}$.
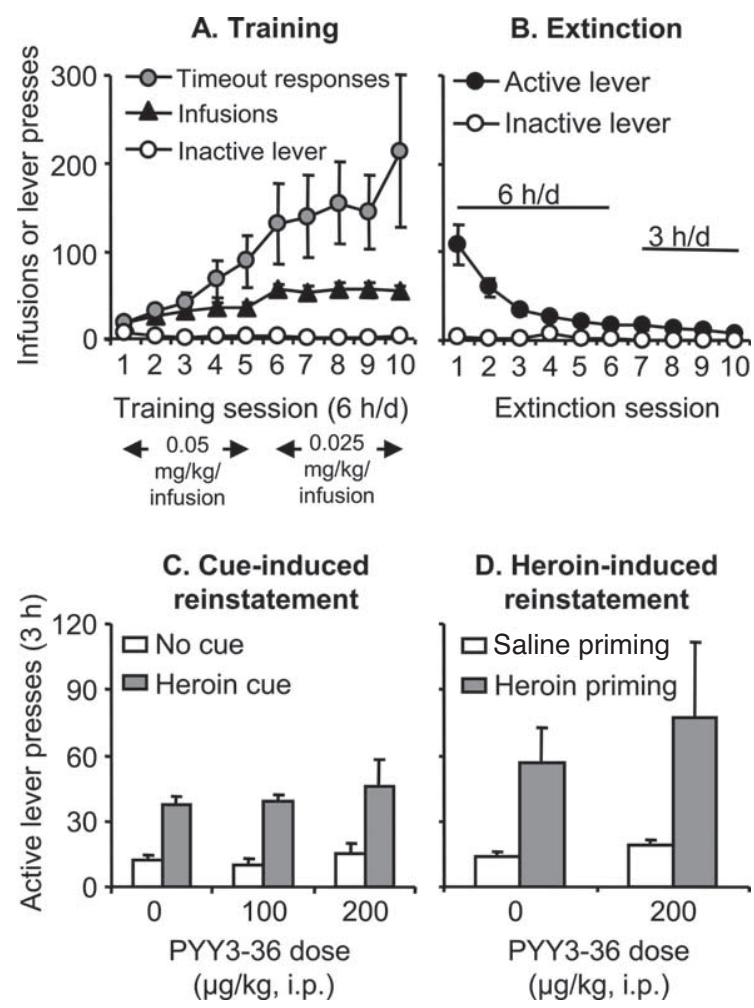

Figure 7. PYY3-36 systemic injections have no effect on cue-induced and heroin priminginduced reinstatement of heroin seeking. $\boldsymbol{A}$, Training. Mean \pm SEM number of infusions, timeout responses, and inactive lever presses over the 10 training days (fixed-ratio- $140 \mathrm{~s}$ timeout reinforcement schedule; $n=19$ ). $\boldsymbol{B}$, Extinction. Mean number of presses on the previously active or inactive lever during the extinction sessions. Lever pressing in the absence of the $5 \mathrm{~s}$ tone-light cue was extinguished over consecutive days for $6 \mathrm{~h}$ per session on days $1-6$ and for $3 \mathrm{~h}$ for the subsequent sessions. C, Heroin cue. Mean number of active lever presses in the no-cue (extinction) and the heroin-cue conditions in rats given injections of vehicle or PYY3-3630 min before the test sessions ( $n=9-10$ for each PYY3-36 dose; $n=19$ for the vehicle condition). During testing, lever presses led to contingent presentation of the $5 s$ tone light cue. $D$, Heroin priming. Mean number of active lever presses in the vehicle (saline) and heroin-priming $(0.25$ $\mathrm{mg} / \mathrm{kg}$ ) conditions in rats given injections of the PYY3-36 vehicle $(n=4)$ or PYY3-36 $(n=5) 30$ $\mathrm{min}$ before the test sessions. Saline (subcutaneously) or heroin was injected $5 \mathrm{~min}$ before the start of the session.

\section{Neuronal mechanisms underlying PYY3-36 effects on} reinstatement of food seeking

In initial anatomical characterization of the inhibitory effect of systemic PYY3-36 on reinstatement of food seeking, we exam- ined the role of Arc because Arc PYY3-36 injections inhibit feeding (Batterham et al., 2002) and Arc BIIE0246 injections reverse the inhibitory effect of systemic PYY3-36 on home-cage feeding (Abbott et al., 2005). Our data clearly indicate that Arc does not mediate PYY3-36 systemic effects on pellet cue-induced reinstatement. We also interpret our data to suggest that the PYY3-36 Y2 receptor-dependent systemic effect on pellet priminginduced reinstatement does not involve the Arc. Although Arc PYY3-36 injections decreased pellet priming-induced reinstatement, the systemic effect of PYY3-36 on this reinstatement was reversed by systemic but not Arc injections of BIIE0246. It is unlikely that the lack of effect of Arc BIIE0246 injections on inhibition of pellet priming-induced reinstatement by systemic PYY3-36 is attributable to the intracranial dose of BII0246 used here (0.5 $\mu \mathrm{g}$ per side); at this dose, Arc BIIE0246 injections increased $(>250 \%)$ feeding in food-sated rats and reversed PYY3-36 systemic effects on home-cage feeding (Abbott et al., 2005).

The site of action of PYY3-36 Y2 receptor-dependent systemic effects on reinstatement of food seeking is unknown. It is unlikely that PYY3-36 peripheral effects mediate conditioned resumption of lever presses induced by acute exposure to food taste (pellet priming) or the tone-light pellet cue, because under our conditions, PYY3-36 had minimal effects on food self-administration. PYY3-36 can reach multiple brain sites after systemic injections (Nonaka et al., 2003) and activate Y2 receptors expressed in hypothalamic nuclei other than the Arc, including lateral hypothalamus and anterior hypothalamic nucleus (Fetissov et al., 2004). Thus, systemic PYY3-36 effects on these hypothalamic sites may mediate its effect on reinstatement of food seeking. Y2 mRNA is also detected in many extrahypothalamic sites (Parker and Herzog, 1999). Stanic et al. (2006) reported that Y2 receptors are expressed at low to moderate levels in extrahypothalamic sites involved in food reward (Kelley and Berridge, 2002; Wise, 2006) and reinstatement of drug seeking (Bossert et al., 2005; See, 2005). These areas include ventral tegmental area (VTA), nucleus accumbens core, cingulate cortex, central amygdala, and ventral pallidum (Stanic et al., 2006). Thus, PYY3-36 systemic effects on reinstatement may be mediated by these extrahypothalamic sites. In a preliminary study, we found that VTA PYY3-36 injections had no effect on pellet priming-induced reinstatement [mean \pm SEM for the vehicle and PYY3-36 (0.4 $\mu$ g per side) were $34 \pm 12$ and $51 \pm 14$, respectively]. The role of other mesocorticolimbic sites that express Y2 receptors in the systemic effect of PYY3-36 on reinstatement of food seeking is currently unknown.

An intriguing finding in our report was that PYY3-36 Arc injections decreased pellet priming- but not pellet cue-induced reinstatement of food seeking. A potential mechanism for the local effect of PYY3-36 on pellet priming-induced reinstatement is inhibition of $\beta$-endorphin projections from Arc to nucleus accumbens (Finley et al., 1981; Akil et al., 1984). Local PYY3-36 application inhibits Arc pro-opiomelanocortin (the precursor for $\beta$-endorphin) neuronal activity (Acuna-Goycolea and van den Pol, 2005). In the context of food-taking behavior, the Arcaccumbens $\beta$-endorphin projection is of interest, because stimulation of accumbens $\mu$-opioid receptors (the main receptor target of Arc-accumbens $\beta$-endorphin neurons) increases foodtaking behavior and food palatability (Zhang et al., 1998; Pecina and Berridge, 2000; Kelley and Berridge, 2002). Published data on the effect of food-taking behavior on $\beta$-endorphin release in accumbens are not available. However, there is evidence that $\beta$-endorphin accumbens levels are increased during reward 
(brain stimulation, heroin, cocaine) seeking, as measured in extinction tests (Roth-Deri et al., 2003; Zangen and Shalev, 2003).

\section{Different effects of PYY3-36 on food- and drug-taking behaviors}

Our results revealed several dissociations in PYY3-36 behavioral effects. The first is that PYY3-36, at doses that had minimal effects on ongoing food self-administration, inhibited pellet primingand pellet cue-induced reinstatement. The self-administration procedure measures the operant reinforcing effects of unconditioned stimuli such as food and drugs (Schuster and Thompson, 1969; Wise, 1989). Thus, our data suggest that mechanisms underlying food reinforcement differ from those underlying food relapse. This notion is in agreement with findings that pharmacological agents that attenuate cue-induced reinstatement of food seeking have no effect on ongoing food self-administration (De Vries et al., 2001, 2005; Baptista et al., 2004; Bossert et al., 2006a). Evidence for dissociation between the effects of pharmacological agents on reinstatement versus self-administration is congruent with findings from studies in which drug selfadministration and reinstatement models were used to assess mechanisms underlying drug reinforcement and relapse (De Vries and Shippenberg, 2002; Shalev et al., 2002; Kalivas and Volkow, 2005).

The second dissociation in PYY3-36 effects concerns its selective attenuation of pellet priming- and pellet cue-induced reinstatement but not yohimbine-induced reinstatement. It is unlikely that these results are attributable to different pretreatment times (30 min for cue and priming vs $60 \mathrm{~min}$ for yohimbine): PYY3-36 effects on home-cage feeding can last for at least $8 \mathrm{~h}$ (Batterham et al., 2002; Challis et al., 2003). Our results on the effects of PYY3-36 on pellet priming versus yohimbine are opposite from those we previously reported with the $\mathrm{CRF}_{1}$ receptor antagonist antalarmin, which blocks yohimbine- but not pellet priming-induced reinstatement of food seeking (Ghitza et al., 2006). The different effects of PYY3-36 and antalarmin on reinstatement induced by pellet priming versus yohimbine suggest that different mechanisms underlie relapse induced by food reexposure versus stress. This conclusion is not surprising in light of previous results on dissociations in the effects of pharmacological agents on stress- versus drug priming- or drug cue-induced reinstatement (Shaham et al., 2000; Le and Shaham, 2002; Lu et al., 2003; Weiss, 2005).

The third dissociation concerns the effect of PYY3-36 on reinstatement of food seeking versus heroin seeking. Doses of PYY3-36 that inhibited pellet cue- and pellet priming-induced reinstatement had no effect on heroin cue- and heroin priminginduced reinstatement. Although there are several reports that pharmacological and neuroanatomical manipulations that inhibit reinstatement of drug seeking have no effect on reinstatement of food seeking (Kalivas and McFarland, 2003; Schmidt et al., 2005; See, 2005), there are no published reports that a pharmacological manipulation can selectively inhibit reinstatement of food but not drug seeking. One issue to consider here is that although the cue-induced reinstatement manipulation is similar for both food and heroin, this is not the case for the priming manipulation. For food, noncontingent delivery of one $45 \mathrm{mg}$ pellet likely induces reinstatement, because of the taste cue properties of the pellet, whereas for heroin priming, reinstatement is attributable to drug effects on brain opiate receptors (Stewart, 1984).

\section{Methodological considerations}

We interpret our data to suggest that systemic PYY3-36 injections decreased the rat's motivation to seek high-fat food after exposure to pellet priming or pellet cues. However, alternative interpretations should be considered. PYY3-36 injections had minimal effects on high rates of responding for food pellets (Fig. 2), making it unlikely that PYY3-36 inhibited reinstatement of food seeking because it disrupted motor performance or induced conditioned taste aversion (Halatchev and Cone, 2005). Blockade of Y2 receptors induces anxiolytic-like effects in rodents (Heilig, 2004). Thus, PYY3-36 (Y2 receptor agonist) effects on reinstatement may be attributable to induction of anxiety-like states that can inhibit operant responding (Geller et al., 1962). This interpretation is unlikely, because in our model yohimbine (a classical anxiogenic drug) potently reinstates food seeking (Fig. 6).

An issue to consider is the anatomical specificity of the effect of Arc PYY3-36 injections on pellet priming-induced reinstatement. Drugs injected into specific brain sites can change behavior by diffusing away from the injection site into either nearby or distal sites (if diffuse into ventricles) (Johnson and Epstein, 1975; Wise and Hoffman, 1992). Our results, however, suggest that the effect of Arc PYY3-36 injections on reinstatement is not attributable to injection site diffusion: missed injections, primarily found in VMH were ineffective (see Results).

Finally, it is unlikely that the different effects of PYY3-36 on reinstatement of food seeking versus heroin seeking are attributable to differences in response rates during training (Fig. $1 B$ vs Fig. 7A). There is little evidence from drug reinstatement studies that different response rates during training qualitatively influence the effect of pharmacological agents on reinstatement after extinction (Shalev et al., 2002).

\section{Concluding remarks}

We identified a novel and potent effect of PYY3-36 on relapse to high-fat food seeking, as measured in the reinstatement procedure. To the degree that this procedure is a valid relapse model (Self and Nestler, 1998; Shaham et al., 2003; Epstein et al., 2006), our findings may have implications for relapse prevention during dieting. There are conflicting reports on PYY3-36 effect on home-cage feeding (Boggiano et al., 2005), and we found that PYY3-36 had weak effects on high-fat food self-administration. Based on these results, our reinstatement data suggest that PYY3-36 may be more effective for the treatment of relapse to maladaptive high-fat food-seeking habits during dieting than for reducing ongoing high-fat food consumption.

\section{References}

Abbott CR, Small CJ, Kennedy AR, Neary NM, Sajedi A, Ghatei MA, Bloom SR (2005) Blockade of the neuropeptide Y Y2 receptor with the specific antagonist BIIE0246 attenuates the effect of endogenous and exogenous peptide YY(3-36) on food intake. Brain Res 1043:139-144.

Abizaid A, Gao Q, Horvath TL (2006) Thoughts for food: brain mechanisms and peripheral energy balance. Neuron 51:691-702.

Acuna-Goycolea C, van den Pol AN (2005) Peptide $Y_{3-36}$ inhibits both anorexigenic proopiomelanocortin and orexigenic neuropeptide $\mathrm{Y}$ neurons: implications for hypothalamic regulation of energy homeostasis. J Neurosci 25:10510-10519.

Akil H, Watson SJ, Young E, Lewis ME, Khachaturian H, Walker JM (1984) Endogenous opioids: biology and function. Annu Rev Neurosci 7:223-255.

Avena NM, Rada P, Hoebel BG (2007) Evidence for sugar addiction: behavioral and neurochemical effects of intermittent, excessive sugar intake. Neurosci Biobehav Rev, in press.

Ballantyne GH (2006) Peptide YY(1-36) and peptide YY(3-36): part I. Distribution, release and actions. Obes Surg 16:651-658. 
Baptista MA, Martin-Fardon R, Weiss F (2004) Preferential effects of the metabotropic glutamate $2 / 3$ receptor agonist LY379268 on conditioned reinstatement versus primary reinforcement: comparison between cocaine and a potent conventional reinforcer. J Neurosci 24:4723-4727.

Batterham RL, Cowley MA, Small CJ, Herzog H, Cohen MA, Dakin CL, Wren AM, Brynes AE, Low MJ, Ghatei MA, Cone RD, Bloom SR (2002) Gut hormone PYY(3-36) physiologically inhibits food intake. Nature 418:650-654

Batterham RL, Cohen MA, Ellis SM, Le Roux CW, Withers DJ, Frost GS, Ghatei MA, Bloom SR (2003) Inhibition of food intake in obese subjects by peptide YY3-36. N Engl J Med 349:941-948.

Boggiano MM, Chandler PC, Oswald KD, Rodgers RJ, Blundell JE, Ishii Y, Beattie AH, Holch P, Allison DB, Schindler M, Arndt K, Rudolf K, Mark M, Schoelch C, Joost HG, Klaus S, Thone-Reineke C, Benoit SC, Seeley RJ, Beck-Sickinger AG, et al. (2005) PYY3-36 as an anti-obesity drug target. Obes Rev 6:307-322.

Bossert JM, Liu SY, Lu L, Shaham Y (2004) A role of ventral tegmental area glutamate in contextual cue-induced relapse to heroin seeking. J Neurosci 24:10726-10730.

Bossert JM, Ghitza UE, Lu L, Epstein DH, Shaham Y (2005) Neurobiology of relapse to heroin and cocaine seeking: an update and clinical implications. Eur J Pharmacol 526:36-50.

Bossert JM, Poles GC, Sheffler-Collins SI, Ghitza UE (2006a) The mGluR2/3 agonist LY379268 attenuates context- and discrete cueinduced reinstatement of sucrose seeking but not sucrose selfadministration in rats. Behav Brain Res 173:148-152.

Bossert JM, Gray SM, Lu L, Shaham Y (2006b) Activation of group II metabotropic glutamate receptors in the nucleus accumbens shell attenuates context-induced relapse to heroin seeking. Neuropsychopharmacology 31:2197-2209.

Bremner JD, Krystal JH, Southwick SM, Charney DS (1996a) Noradrenergic mechanisms in stress and anxiety: I. preclinical studies. Synapse 23:28-38.

Bremner JD, Krystal JH, Southwick SM, Charney DS (1996b) Noradrenergic mechanisms in stress and anxiety: II. clinical studies. Synapse 23:39-51.

Carr KD (2002) Augmentation of drug reward by chronic food restriction: behavioral evidence and underlying mechanisms. Physiol Behav 76:353-564.

Challis BG, Pinnock SB, Coll AP, Carter RN, Dickson SL, O’Rahilly S (2003) Acute effects of PYY3-36 on food intake and hypothalamic neuropeptide expression in the mouse. Biochem Biophys Res Commun 311:915-919.

Chelikani PK, Haver AC, Reeve Jr JR, Keire DA, Reidelberger RD (2006) Daily, intermittent intravenous infusion of peptide YY(3-36) reduces daily food intake and adiposity in rats. Am J Physiol Regul Integr Comp Physiol 290:R298-R305.

Colantuoni C, Rada P, McCarthy J, Patten C, Avena NM, Chadeayne A, Hoebel BG (2002) Evidence that intermittent, excessive sugar intake causes endogenous opioid dependence. Obes Res 10:478-488.

Corwin RL, Buda-Levin A (2004) Behavioral models of binge-type eating. Physiol Behav 82:123-130.

De Vries TJ, Schoffelmeer AN (2005) Cannabinoid CB1 receptors control conditioned drug seeking. Trends Pharmacol Sci 26:420-426.

De Vries TJ, Shippenberg TS (2002) Neural systems underlying opiate addiction. J Neurosci 22:3321-3325.

De Vries TJ, Shaham Y, Homberg JR, Crombag H, Schuurman K, Dieben J, Vanderschuren LJ, Schoffelmeer AN (2001) A cannabinoid mechanism in relapse to cocaine seeking. Nat Med 7:1151-1154.

De Vries TJ, de Vries W, Janssen MC, Schoffelmeer AN (2005) Suppression of conditioned nicotine and sucrose seeking by the cannabinoid-1 receptor antagonist SR141716A. Behav Brain Res 161:164-168.

DiLeone RJ, Georgescu D, Nestler EJ (2003) Lateral hypothalamic neuropeptides in reward and drug addiction. Life Sci 73:759-768.

Doods H, Gaida W, Wieland HA, Dollinger H, Schnorrenberg G, Esser F, Engel W, Eberlein W, Rudolf K (1999) BIIE0246: a selective and high affinity neuropeptide Y Y(2) receptor antagonist. Eur J Pharmacol 384:R3-R5.

Drewnowski A (1997) Taste preferences and food intake. Annu Rev Nutr 17:237-253.

Duarte C, Biala G, Le Bihan C, Hamon M, Thiebot MH (2003) Respective roles of dopamine $\mathrm{D} 2$ and $\mathrm{D} 3$ receptors in food-seeking behaviour in rats. Psychopharmacology (Berl) 166:19-32.
Eberlein GA, Eysselein VE, Schaeffer M, Layer P, Grandt D, Goebell H, Niebel W, Davis M, Lee TD, Shively JE, Reeve Jr JR (1989) A new molecular form of PYY: structural characterization of human PYY(3-36) and PYY(1-36). Peptides 10:797-803.

Epstein DH, Preston KL, Stewart J, Shaham Y (2006) Toward a model of drug relapse: an assessment of the validity of the reinstatement procedure. Psychopharmacology 189:1-16.

Fetissov SO, Kopp J, Hokfelt T (2004) Distribution of NPY receptors in the hypothalamus. Neuropeptides 38:175-188.

Finley JC, Lindstrom P, Petrusz P (1981) Immunocytochemical localization of beta-endorphin-containing neurons in the rat brain. Neuroendocrinology 33:28-42.

Geller I, Kulak Jr JT, Seifter J (1962) The effects of chlordiazepoxide and chlorpromazine on a punishment discrimination. Psychopharmacologia 3:374-785

Ghitza UE, Gray SM, Epstein DH, Rice KC, Shaham Y (2006) The anxiogenic drug yohimbine reinstates palatable food seeking in a rat relapse model: a role of $\mathrm{CRF}(1)$ receptors. Neuropsychopharmacology 31:2188-2196.

Grandt D, Teyssen S, Schimiczek M, Reeve Jr JR, Feth F, Rascher W, Hirche H, Singer MV, Layer P, Goebell H, Ho FJ, Eysselein UE (1992) Novel generation of hormone receptor specificity by amino terminal processing of peptide YY. Biochem Biophys Res Commun 186:1299-1306.

Grilo CM, Shiffman S, Wing RR (1989) Relapse crises and coping among dieters. J Consult Clin Psychol 57:488-495.

Hagan MM, Wauford PK, Chandler PC, Jarrett LA, Rybak RJ, Blackburn K (2002) A new animal model of binge eating: key synergistic role of past caloric restriction and stress. Physiol Behav 77:45-54.

Hagan MM, Chandler PC, Wauford PK, Rybak RJ, Oswald KD (2003) The role of palatable food and hunger as trigger factors in an animal model of stress induced binge eating. Int J Eat Disord 34:183-197.

Halatchev IG, Cone RD (2005) Peripheral administration of PYY(3-36) produces conditioned taste aversion in mice. Cell Metab 1:159-168.

Heilig M (2004) The NPY system in stress, anxiety and depression. Neuropeptides 38:213-224.

Herman CP, Polivy J (1975) Anxiety, restraint, and eating behavior. J Abnorm Psychol 84:66-72.

Horvitz JC, Ettenberg A (1988) Haloperidol blocks the response-reinstating effects of food reward: a methodology for separating neuroleptic effects on reinforcement and motor processes. Pharmacol Biochem Behav 31:861-865.

Johnson AK, Epstein AN (1975) The cerebral ventricles as the avenue for the dipsogenic action of intracranial angiotensin. Brain Res 86:399-418.

Kalivas PW, McFarland K (2003) Brain circuitry and the reinstatement of cocaine-seeking behavior. Psychopharmacology 168:44-56.

Kalivas PW, Volkow ND (2005) The neural basis of addiction: a pathology of motivation and choice. Am J Psychiatry 162:1403-1413.

Kelley AE, Berridge KC (2002) The neuroscience of natural rewards: relevance to addictive drugs. J Neurosci 22:3306-3311.

Larhammar D, Salaneck E (2004) Molecular evolution of NPY receptor subtypes. Neuropeptides 38:141-151.

Le A, Shaham Y (2002) Neurobiology of relapse to alcohol in rats. Pharmacol Ther 94:137-156.

Le AD, Harding S, Juzytsch W, Funk D, Shaham Y (2005) Role of alpha-2 adrenoceptors in stress-induced reinstatement of alcohol seeking and alcohol self-administration in rats. Psychopharmacology 179:366-373.

Lee B, Tiefenbacher S, Platt DM, Spealman RD (2004) Pharmacological blockade of alpha(2)-arenoceptors induces reinstatement of cocaineseeking behavior in squirrel monkeys. Neuropsychopharmacology 29:686-693.

Leibowitz SF (1995) Brain peptides and obesity: pharmacologic treatment. Obes Res 3 [Suppl 4]:573S-589S.

Lu L, Shepard JD, Scott Hall F, Shaham Y (2003) Effect of environmental stressors on opiate and psychostimulant reinforcement, reinstatement and discrimination in rats: a review. Neurosci Biobehav Rev 27:457-491.

Moran TH, Smedh U, Kinzig KP, Scott KA, Knipp S, Ladenheim EE (2005) Peptide YY(3-36) inhibits gastric emptying and produces acute reductions in food intake in rhesus monkeys. Am J Physiol Regul Integr Comp Physiol 288:R384-R388.

Murphy KG, Dhillo WS, Bloom SR (2006) Gut peptides in the regulation of food intake and energy homeostasis. Endocr Rev 27:719-727.

Nair SG, Gray SM, Ghitza UE (2006) Role of food type in yohimbine- and 
pellet-priming-induced reinstatement of food seeking. Physiol Behav $88: 559-566$.

Nonaka N, Shioda S, Niehoff ML, Banks WA (2003) Characterization of blood-brain barrier permeability to PYY3-36 in the mouse. J Pharmacol Exp Ther 306:948-953.

Parker RM, Herzog H (1999) Regional distribution of Y-receptor subtype mRNAs in rat brain. Eur J Neurosci 11:1431-1448.

Paxinos G, Watson C (2005) The rat brain in stereotaxic coordinates, Ed 5. San Diego: Elsevier.

Pecina S, Berridge KC (2000) Opioid site in nucleus accumbens shell mediates eating and hedonic "liking" for food: map based on microinjection Fos plumes. Brain Res 863:71-86.

Peterson CB, Mitchell JE (1999) Psychosocial and pharmacological treatment of eating disorders: a review of research findings. J Clin Psychol 55:685-697.

Renshaw D, Batterham RL (2005) Peptide YY: a potential therapy for obesity. Curr Drug Targets 6:171-179.

Roth-Deri I, Zangen A, Aleli M, Goelman RG, Pelled G, Nakash R, GispanHerman I, Green T, Shaham Y, Yadid G (2003) Effect of experimenterdelivered and self-administered cocaine on extracellular beta-endorphin levels in the nucleus accumbens. J Neurochem 84:930-938.

Schmidt HD, Anderson SM, Famous KR, Kumaresan V, Pierce RC (2005) Anatomy and pharmacology of cocaine priming-induced reinstatement of drug seeking. Eur J Pharmacol 526:65-76.

Schuster CR, Thompson T (1969) Self administration of and behavioral dependence on drugs. Annu Rev Pharmacol 9:483-502.

Scott V, Kimura N, Stark JA, Luckman SM (2005) Intravenous peptide YY3-36 and Y2 receptor antagonism in the rat: effects on feeding behaviour. J Neuroendocrinol 17:452-457.

See RE (2005) Neural substrates of cocaine-cue associations that trigger relapse. Eur J Pharmacol 526:140-146.

Self DW, Nestler EJ (1998) Relapse to drug-seeking: neural and molecular mechanisms. Drug Alcohol Depend 51:49-69.

Shaham Y, Rajabi H, Stewart J (1996) Relapse to heroin-seeking under opioid maintenance: the effects of opioid withdrawal, heroin priming and stress. J Neurosci 16:1957-1963.

Shaham Y, Funk D, Erb S, Brown TJ, Walker CD, Stewart J (1997) Corticotropin-releasing factor, but not corticosterone, is involved in stress-induced relapse to heroin-seeking in rats. J Neurosci 17:2605-2614.

Shaham Y, Erb S, Stewart J (2000) Stress-induced relapse to heroin and cocaine seeking in rats: a review. Brain Res Brain Res Rev 33:13-33.

Shaham Y, Shalev U, Lu L, De Wit H, Stewart J (2003) The reinstatement model of drug relapse: history, methodology and major findings. Psychopharmacology 168:3-20.

Shalev U, Yap J, Shaham Y (2001a) Leptin attenuates food deprivationinduced relapse to heroin seeking. J Neurosci 21:RC129(1-5).

Shalev U, Morales M, Hope B, Yap J, Shaham Y (2001b) Time-dependent changes in extinction behavior and stress-induced reinstatement of drug seeking following withdrawal from heroin in rats. Psychopharmacology 156:98-107.

Shalev U, Grimm JW, Shaham Y (2002) Neurobiology of relapse to heroin and cocaine seeking: a review. Pharmacol Rev 54:1-42.

Shepard JD, Bossert JM, Liu SY, Shaham Y (2004) The anxiogenic drug yohimbine reinstates methamphetamine seeking in a rat model of drug relapse. Biol Psychiatry 55:1082-1089.

Stanic D, Brumovsky P, Fetissov S, Shuster S, Herzog H, Hokfelt T (2006) Characterization of neuropeptide $\mathrm{Y} 2$ receptor protein expression in the mouse brain. I. Distribution in cell bodies and nerve terminals. J Comp Neurol 499:357-390.

Stewart J (1984) Reinstatement of heroin and cocaine self-administration behavior in the rat by intracerebral application of morphine in the ventral tegmental area. Pharmacol Biochem Behav 20:917-923.

Tatemoto K, Mutt V (1980) Isolation of two novel candidate hormones using a chemical method for finding naturally occurring polypeptides. Nature 285:417-418.

Tschop M, Castaneda TR, Joost HG, Thone-Reineke C, Ortmann S, Klaus S, Hagan MM, Chandler PC, Oswald KD, Benoit SC, Seeley RJ, Kinzig KP, Moran TH, Beck-Sickinger AG, Koglin N, Rodgers RJ, Blundell JE, Ishii Y, Beattie AH, Holch P, et al. (2004) Physiology: does gut hormone PYY3-36 decrease food intake in rodents? Nature 430:1 p following 165; discussion $2 \mathrm{p}$ following 165 .

Wahlestedt C, Yanaihara N, Hakanson R (1986) Evidence for different preand post-junctional receptors for neuropeptide $\mathrm{Y}$ and related peptides. Regul Pept 13:307-318.

Weiss F (2005) Neurobiology of craving, conditioned reward and relapse. Curr Opin Pharmacol 5:9-19.

Wise RA (1989) The brain and reward. In: The neurophamracological basis of reward (Liebman JM, Kooper SJ, eds), pp 377-424. Oxford: Oxford UP.

Wise RA (2006) Role of brain dopamine in food reward and reinforcement. Philos Trans R Soc Lond B Biol Sci 361:1149-1458.

Wise RA, Hoffman DC (1992) Localization of drug reward mechanisms by intracranial injections. Synapse 10:247-263.

Yokel RA (1987) Intravenous self-administration: response rates, the effects of pharmacological challenges, and drug preference. In: Methods of assessing the reinforcing properties of abused drugs (Bozarth MA, ed), pp 1-34. New York: Springer.

Zangen A, Shalev U (2003) Nucleus accumbens beta-endorphin levels are not elevated by brain stimulation reward but do increase with extinction. Eur J Neurosci 17:1067-1072.

Zhang M, Gosnell BA, Kelley AE (1998) Intake of high-fat food is selectively enhanced by mu opioid receptor stimulation within the nucleus accumbens. J Pharmacol Exp Ther 285:908-914. 